\title{
Development and validation of a novel nomogram for predicting distant metastasis-free survival among breast cancer patients
}

\author{
Yan Wang ${ }^{1,2 \#}$, Yaping Yang ${ }^{1,2 \#}$, Zhengbo Chen $^{3 \#}$, Teng Zhu ${ }^{4}$, Jiannan Wu ${ }^{1,2}$, Fengxi Su ${ }^{1,2}$, Heran Deng ${ }^{1,2}$ \\ ${ }^{1}$ Guangdong Provincial Key Laboratory of Malignant Tumor Epigenetics and Gene Regulation, Sun Yat-sen Memorial Hospital, Sun Yat-sen \\ University, Guangzhou 510120, China; ${ }^{2}$ Department of Breast Surgery, Breast Tumor Center, Sun Yat-sen Memorial Hospital, Sun Yat-sen \\ University, Guangzhou 510120, China; ${ }^{3}$ Department of General Surgery,Guangdong General Hospital, Guangdong Academy of Medical Sciences, \\ Guangzhou 510080, China; ${ }^{4}$ Department of Breast Cancer, Cancer Center, Guangdong General Hospital, Guangdong Academy of Medical \\ Sciences, Guangzhou 510080, China \\ Contributions: (I) Conception and design: H Deng, F Su, J Wu, Y Wang; (II) Administrative support: F Su, J Wu; (III) Provision of study materials \\ or patients: Y Wang, Y Yang, Z Chen, T Zhu; (IV) Collection and assembly of data: Y Wang, Y Yang, Z Chen, T Zhu; (V) Data analysis and \\ interpretation: Y Wang, Y Yang, Z Chen; (VI) Manuscript writing: All authors; (VII) Final approval of manuscript: All authors. \\ "These authors contributed equally to this work. \\ Correspondence to: Heran Deng; Fengxi Su; Jiannan Wu. Breast Tumor Center, Sun Yat-sen Memorial Hospital, Sun Yat-sen University, 107 Yanjiang \\ West Road, Guangzhou 510120, China. Email: dengheran0930@sina.com; fengxisu@vip.163.com; wujiann3@mail.sysu.edu.cn.
}

\begin{abstract}
Background: Distant metastasis (DM) from breast cancer has a poor prognosis. Our objective was to develop and validate a nomogram to predict individual distant metastasis-free survival (DMFS) and risk stratification in non-metastatic breast cancer patients.

Methods: A nomogram was based on an analysis of 1,201 breast cancer patients treated at Sun Yat-sen Memorial Hospital from 2001 to 2014. Using univariate and multivariate analyses to identify the predictors, this model was externally validated in an independent cohort of 538 patients from the Guangdong General Hospital between 2004 and 2012. The predictive discrimination and calibration ability of this nomogram were assessed using concordance index (C-index), risk group stratification, and calibration curve.

Results: The 5-year DMFS in the training and validation cohorts were $95.74 \%$ and $91.02 \%$, respectively. On multivariable analysis of training cohort, the prognostic factors in the nomogram comprised age, tumor size, lymph node status, molecular subtype, and lymphovascular invasion (LVI). The C-index of our model was 0.75 [95\% confidence interval (CI): 0.67-0.83] for the training cohort and 0.71 (95\% CI: 0.64-0.78) for the validation cohort. The calibration curves for 5-year DMFS showed good agreement between the model prediction and actual observation. Based on the risk stratification, Kaplan-Meier curves indicated that the low-risk group had significantly better prognosis than the high-risk group $(\mathrm{P}<0.001)$.

Conclusions: Our nomogram can provide an individual prediction of 5-year DMFS in non-metastatic breast cancer patients. This prognostic tool may help clinicians to make appropriate treatment regimens and optimal surveillance plans.
\end{abstract}

Keywords: Breast cancer; nomogram; distant metastasis-free survival (DMFS)

Submitted Sep 24, 2019. Accepted for publication Sep 29, 2019.

doi: $10.21037 /$ atm.2019.10.10

View this article at: http://dx.doi.org/10.21037/atm.2019.10.10

\section{Introduction}

Breast cancer is the most frequent cancer among women in the world. In China, breast cancer is an enormous public health problem and has become the sixth leading cause of death from cancer (1). With the great advances in cancer treatments, the prognosis of early breast cancer patients has been improved remarkably. However, approximately $20 \%$ to $25 \%$ of patients with breast cancer will suffer from 
distant metastasis $(\mathrm{DM})$, which is the main cause of breast cancer death (2) .

The heterogeneous nature of breast tumors has led to a diversity of therapeutic strategies and different clinical outcomes. Currently, the American Joint Committee on Cancer (AJCC) tumor-node-metastasis (TNM) staging system is widely used in survival prediction. Patients with non-metastatic breast cancer are stratified depending on tumor size and lymph node involvement. However, the survival outcome of patients with the same stage varies widely (3-5). Once metastasis occurs, the disease is largely incurable, and the median survival of patients ranges from only two to three years (6). Several clinicopathological features such as tumor size, lymph node status, lymphovascular invasion (LVI), estrogen receptor (ER) and progesterone receptor (PR) status, human epidermal growth factor receptor 2 (HER2), and proliferation index (Ki67) expression affect the distant metastasis-free survival (DMFS). Additionally, a combination of these specific biomarkers classified as surrogate molecular subtypes has been proposed to provide biological and prognostic information. Patients with different subtypes of breast cancer have different DMFS. Thus, due to the biological heterogeneity of breast cancer, a useful predictive tool incorporating a growing number of independent risk factors can play an important role in predicting the survival outcomes in breast cancer patients.

Nomograms have been demonstrated to enable a more precise prediction for individual patients in various tumors, including esophageal squamous cell carcinoma, nonsmall cell lung cancer, and ovarian cancer (7-9). Several survival prediction models, such as CancerMath (10) (www. CancerMath.com), PREDICT $(11,12)$ and the ipsilateral breast tumor recurrence program (IBTR) $(13,14)$ are designed to predict survival probability in breast cancer patients. However, these models were established solely based on data from western patients. Chinese women may have different ethnographic features, clinicopathological characteristics, and medical insurance policies. Accurately predicting DMFS for Chinese breast cancer patients may optimize treatment strategies and surveillance plans. However, nomograms for predicting DMFS and risk stratification in non-metastatic breast cancer are scarce. Furthermore, previous predictive models have been mainly based on immunohistochemical biomarkers, but the molecular subtypes of breast cancer have been neglected to some extent.

Therefore, the aim of this study was to use Chinese population databases to develop and validate a prognostic model incorporating molecular subtypes and other important clinicopathological variables for predicting DMFS and risk stratification in breast cancer patients.

\section{Methods}

\section{Patient population}

We selected breast cancer patients from the Sun Yat-sen Memorial Hospital (SYSMH) between 2001 and 2014 as the training cohort. We used the following inclusion criteria: (I) female patients aged 18 years or above; (II) patients who received surgical treatment; and (III) patients with available follow-up data. The exclusion criteria were: (I) patients with metastatic, de novo stage IV breast cancer; (II) patients with phyllode tumors of the breast; (III) patients who received excision before surgery; (IV) patients who received neoadjuvant chemotherapy; and $(\mathrm{V})$ patients who had incomplete clinicopathological data.

An external validation cohort who met the same eligibility criteria was enrolled from the Guangdong General Hospital (GGH) during the period from 2004 to 2012. For eligible patients, the following clinicopathological characteristics were included: age at diagnosis; tumor size; histologic type; postoperative lymph nodal status; Ki67 expression; LVI; ER and PR status; HER2 status; and postoperative chemotherapy. This study was approved by the Institutional Ethics Committees of the SYSMH and GGH (SYSEC-KY-KS-2019-073). Informed consent was waived due to the study's retrospective nature.

\section{Pathological assessment}

All patients received pathological examinations. The ER and PR expression statuses were examined by immunohistochemistry. ER or PR positivity was defined as $1 \%$ or more of positive tumor cells with nuclear staining. Hormone receptor positivity was defined as ER and/or PR positivity. For HER2 status, we used the HercepTest method (15). HER2 positivity was defined as either a score of $3+$ by immunohistochemistry or $2+$ with HER2 amplification via fluorescence in situ hybridization (FISH). HercepTest scores of 0 and 1, or a score of 2 without HER2 amplification via FISH, were considered HER2 negative. The threshold for Ki67 was $20 \%$ (16). Ki67 $\geq 20 \%$ was defined as high Ki67 status, and Ki67 $<20 \%$ was defined as low Ki67 status. The molecular subtypes of breast cancer 
were derived depending on the status of hormone receptor, HER2, and Ki67 as follows: luminal A (hormone receptor positive, HER2 negative, and Ki67 <20\%), luminal B/HER2 negative (hormone receptor positive, HER2 negative, and Ki67 $\geq 20 \%$ ), luminal B/HER2 positive (hormone receptor positive, HER2 positive, and any Ki67), HER2-enriched (ER negative, PR negative, and HER2 positive), and triple negative (ER negative, PR negative, and HER2 negative).

\section{Statistical analysis}

Descriptive analyses of baseline clinicopathological characteristics were performed. Continuous variables were described using the median and range, and categorical variables were described as percentages. DMFS was measured from the date of surgery to the date of DM or the last follow-up. Univariate and multivariate Cox regression were used to assess the prognostic factors. Significant predictors from the multivariate analysis were included in the nomogram development. In addition, the variables that improved the performance of the model were used even if they did not show significance in the multivariate analysis. The discrimination and calibration ability of the model were determined by concordance index (C-index) and calibration curves. Calibration curves measured the agreement between the actual probabilities and the predicted frequencies. We used the median risk score calculated via nomogram as the cutoff point to classify the patients into the low-risk group and the high-risk group. Survival outcomes were evaluated using Kaplan-Meier analyses with the log-rank test. All tests were two-sided, and $\mathrm{P}$ values of 0.05 or less were considered statistically significant. Data analyses were performed using Stata version 13.1 (StataCorp., College Station, TX, USA), and the nomogram was developed using $\mathrm{R}$ (version 2.11.1; R Foundation for Statistical Computing, Vienna, Austria).

\section{Results}

\section{Study population characteristics}

The training cohort included 1,201 non-metastatic breast cancer patients treated at SYSMH with a median follow-up of 57 months. The validation training cohort compromised 538 breast cancer patients in GGH with a median followup of 63 months. The 5 -year DMFS probabilities were 95.74\% [95\% confidence interval (CI): 94.12-96.73\%] in the SYSMH cohort and 91.02\% (95\% CI: 88.25-93.27\%) in the GGH cohort.
Clinicopathological features and treatment patterns of the study population are listed in Table 1. Patients from the two cohorts had a similar age (median age, 48-49 years). In the training cohort, $71.11 \%$ of patients had tumors less than $2 \mathrm{~cm}$, whereas $97.21 \%$ of patients in the validation cohort had tumors less than $2 \mathrm{~cm}$. In the GGH cohort, approximately $60 \%$ of patients had positive lymph node disease. In contrast, approximately $13 \%$ more patients in the training group had positive lymph node disease. In addition, we observed that histologic type, Ki67 status, LVI, and chemotherapy were significantly different in the two cohorts.

\section{Independent prognostic factors for DMFS in the training cobort}

The univariate and multivariate analyses (Table 2) demonstrated that age $\leq 35$ years [vs. age 35-50 years, hazard ratio $(\mathrm{HR})=2.81,95 \% \mathrm{CI}$ : $1.24-6.38, \mathrm{P}=0.014]$, tumor size $>2 \mathrm{~cm}$ (vs. tumor size $\leq 2 \mathrm{~cm}, \mathrm{HR}=2.08,95 \%$ CI: 1.17-3.72, $\mathrm{P}=0.013$ ), positive lymph node involvement (vs. lymph node negative, $\mathrm{HR}=2.81,95 \% \mathrm{CI}: 1.56-5.05, \mathrm{P}=0.001)$, HER2enriched subtype (vs. luminal A, HR $=4.34,95 \%$ CI: 1.36 13.80, $\mathrm{P}=0.013$ ), and triple negative subtype (vs. luminal $\mathrm{A}$, $\mathrm{HR}=3.85$, 95\% CI: $1.19-12.48, \mathrm{P}=0.025$ ) were significantly associated with a poor 5 -year DMFS. The presentation of the LVI was statistically significant in the univariate analysis (vs. LVI negative, HR =2.04, 95\% CI: $1.03-4.03, \mathrm{P}=0.039$ ) but not in the multivariate analysis (vs. LVI negative, HR $=1.61,95 \%$ CI: $0.80-3.25, \mathrm{P}=0.185$ ).

\section{Prognostic nomogram for DMFS}

A nomogram was developed using the significant predictors and LVI, which improved the performance of the nomogram (Figure 1). The C-index was 0.75 (95\% CI: $0.67-0.83)$ for the training cohort and $0.71(95 \%$ CI: $0.64-0.78)$ for the validation cohort. The calibration curves showed that the predicted DMFS agreed well with the actual DMFS in the training cohort and the validation cohort (Figure 2). When applied to the external validation cohort, both the C-index and calibration curves suggested good robustness.

\section{Performance of the nomogram in stratifying patient risk}

According to the points in the training cohort, the median risk score was used to identify the optimal cut-off values. All 
Table 1 Clinicopathologic characteristics of the training cohort and validation cohort

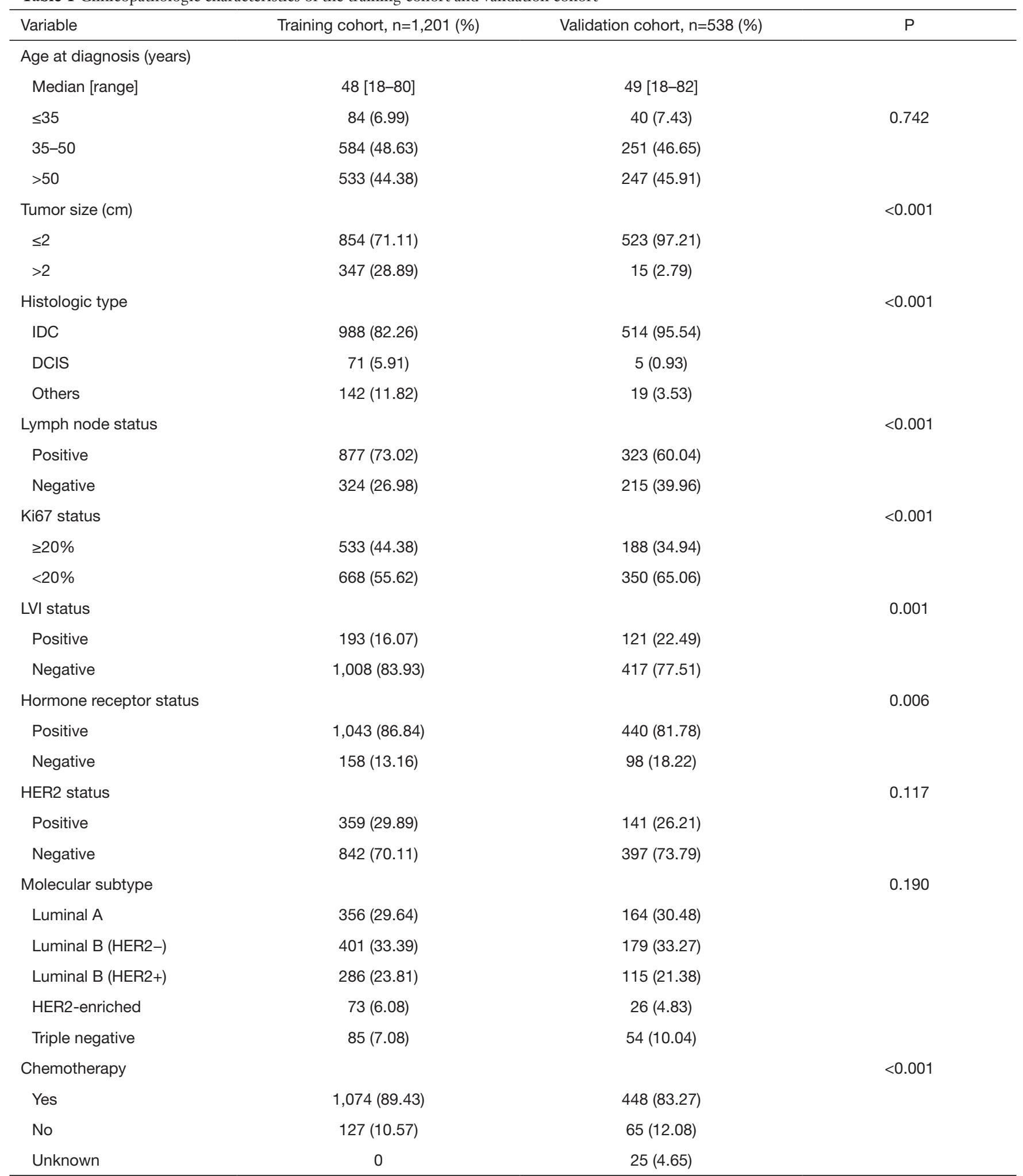

IDC, invasive ductal carcinoma; DCIS, ductal carcinoma in situ; LVI, lymphovascular invasion; HER2, human epidermal growth factor receptor 2 . 
Table 2 Univariate and multivariate Cox proportional hazards regression for DMFS

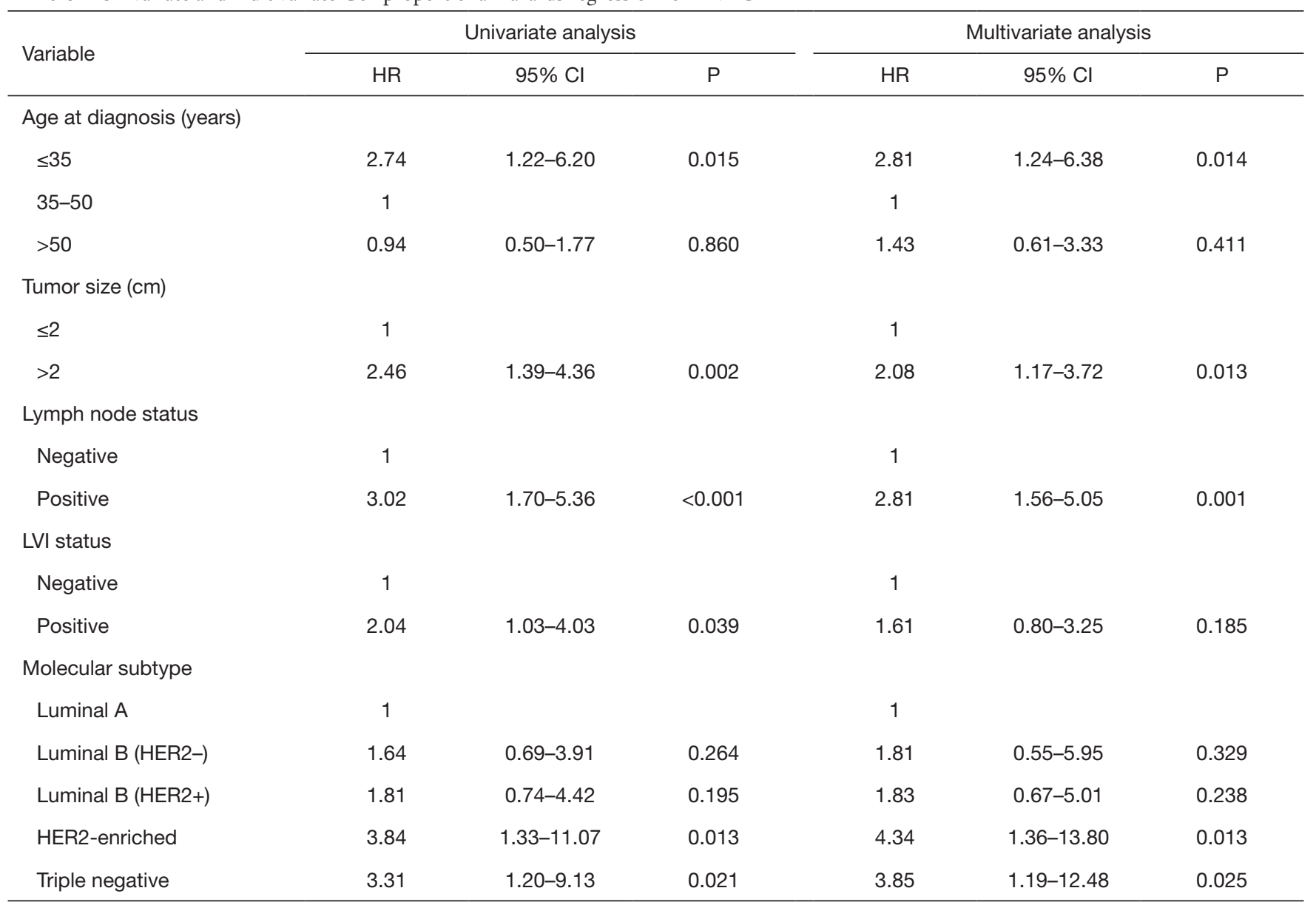

DMFS, distant metastasis-free survival; HR, hazard ratio; Cl, confidence interval; LVI, lymphovascular invasion; HER2, human epidermal growth factor receptor 2 .

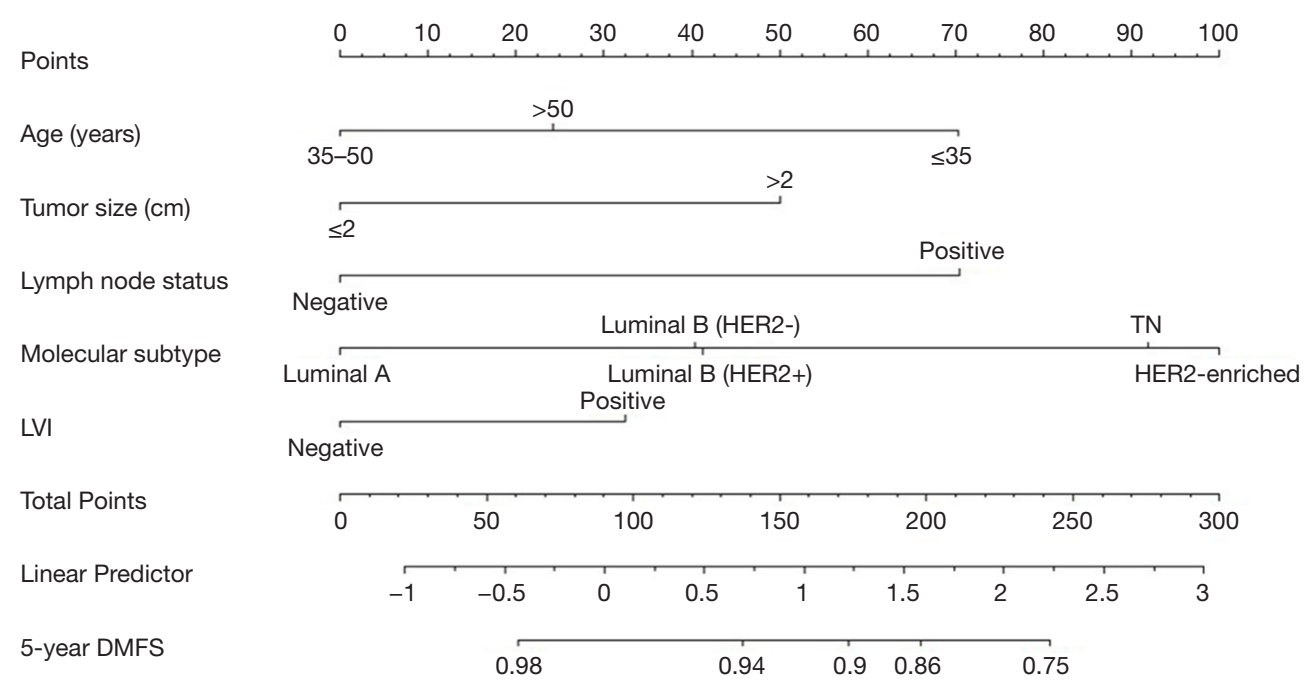

Figure 1 Nomogram for predicting 5-year distant metastasis-free survival in breast cancer patients. 

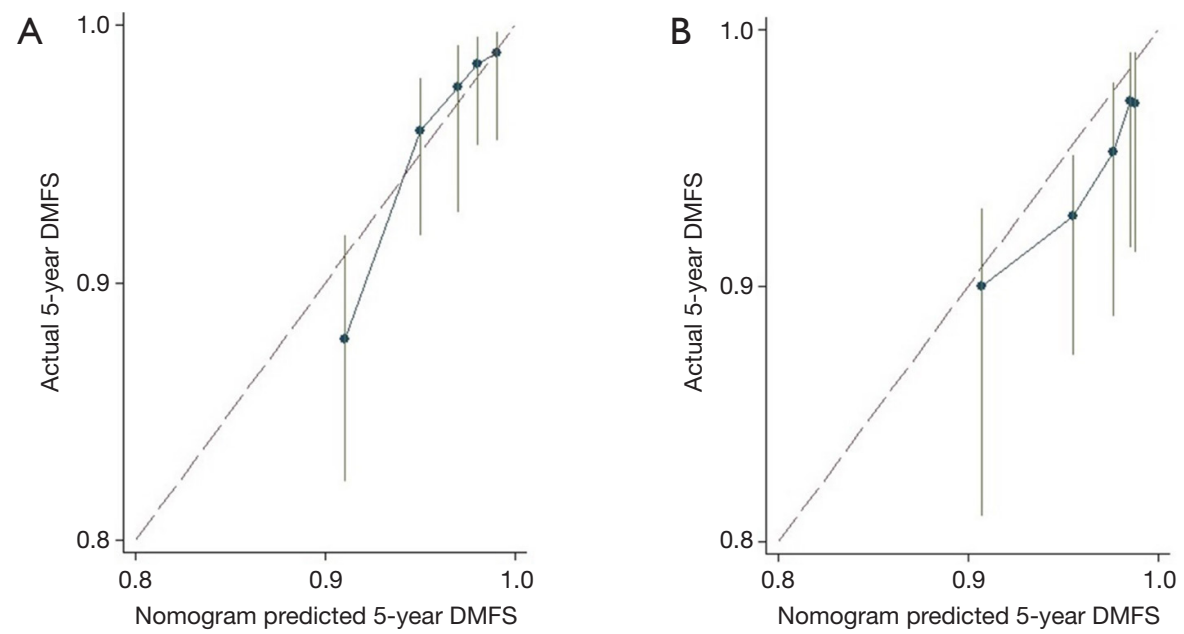

Figure 2 Calibration curves for 5-year distant metastasis-free survival of nomography in the training cohort (A) and validation cohort (B).

A

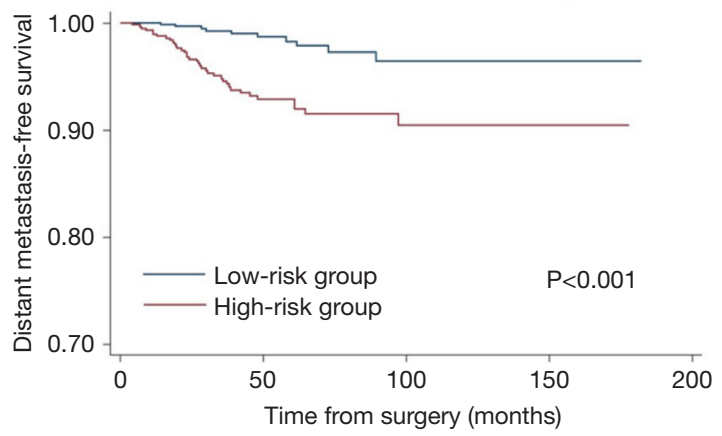

B

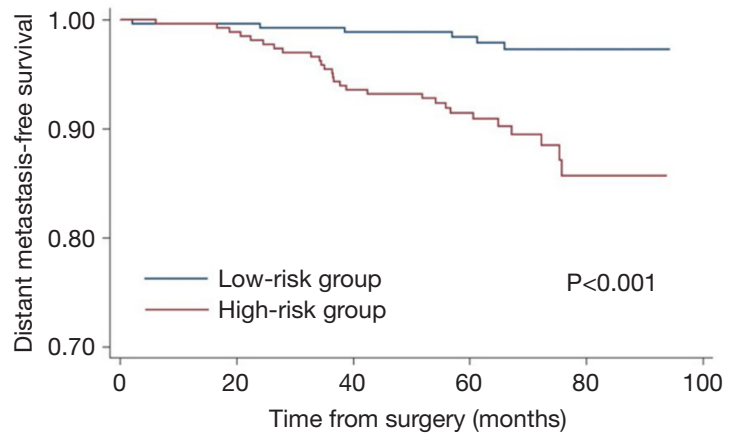

Figure 3 Kaplan-Meier curves for 5 -year distant metastasis-free survival based on risk group stratification of the nomogram in the training cohort (A) and validation cohort (B).

patients were classified into two groups: low risk $(\leq 90.45)$ and high risk (>90.45). In addition, based on the risk stratification, Kaplan-Meier curves for DMFS were used both in the training cohort and the validation cohort. The low-risk group had significantly better DMFS than the high-risk group $(\mathrm{P}<0.001$, Figure 3$)$.

\section{Discussion}

This study was the first to use Chinese databases for developing and externally validating a novel nomogram to predict individualized DMFS for non-metastatic breast cancer patients. Previous nomograms were mainly established based on western women, but Chinese patients have significantly different clinicopathological features to those of patients in western countries. For instance, breast cancer patients in China are considerably younger $(1,17)$, and have more advanced clinical stage at diagnosis due to the lack of screening programs. Furthermore, many new drugs are not included in the reimbursement category due to the imperfect medical insurance system, such as pertuzumab and T-DM1, and the prognosis of breast cancer patients in China is worse than that in the West (1). Therefore, a precise prediction model for the Chinese population is urgently needed.

Many previous nomograms have been constructed with immunohistochemical expression levels to predict the risk of breast cancer metastasis $(18,19)$; however, few models have been constructed based on biological subtypes. Molecular subtypes are known to be of great significance for breast 
cancer treatment patterns, especially for endocrine therapy and targeted therapy. Meanwhile, different molecular subtypes represent different survival outcomes in breast cancer patients (20). As is known, patients with luminal A tumors have better survival outcomes than those with other molecular subtypes. Her2-positive and triple negative breast cancer are associated with shorter DMFS in breast cancer patients. These observations substantiate the assumption that the molecular gene expression specific for a subtype has clinical significance (21). Taken together, we suggest that biological subtypes rather than clinicopathological parameters may be more suitable for our model development in breast cancer patients.

Nearly one quarter of breast cancer patients will present with DM. Because of the heterogeneity of breast cancer, tumor cells can easily metastasize into the bone, lung, liver, brain, and other body parts even after surgery and adjuvant therapy. Patients with DM may suffer from various symptoms, including bone pain, upper abdominal discomfort, cough, headaches etc., which will have a great impact on quality of life. The organs to which breast tumors preferentially metastasize are of clinical and biological importance and are closely associated with patients' prognoses (22). Several studies have suggested that bone metastasis is significantly more likely in breast cancer patients with luminal A and luminal B subtypes than in those with other subtypes (22). Adjuvant therapy with zoledronic acid has been reported to reduce risk of diseasefree survival events in premenopausal and postmenopausal women with breast cancer $(23,24)$, and bisphosphonates not only benefit those who experience bone disease but also those who have an increased risk of bone metastases (25). Based on our study, we could recommend that patients with luminal A or luminal B tumors with a high risk of DM use adjuvant bisphosphonates to improve the survival outcome. Therefore, this prognostic predication contributes to the selection of appropriate local or systemic therapies for highrisk metastatic breast cancer patients.

Additionally, the molecular subtypes are related to distinct patterns of metastatic spread $(26,27)$. A series of studies have demonstrated that luminal/HER2 positive and HER2- enriched breast tumors are related to a notably higher rate of brain, liver, and lung metastases. Triple negative tumors have a high risk of brain and lung metastases (26). As a result, it has been revealed that patients with HER2-enriched or triple negative breast tumors have higher frequencies of central nervous system (CNS) involvement (28-30). Unfortunately, patients who develop
CNS disease from breast cancer have a poor outcome, with a 1 -year survival rate of $20 \%$ (31). A serious consideration is that CNS imaging studies are not conventionally necessary in breast cancer patients with regular follow-up surveillance; therefore, most CNS metastases are detected when symptomatic (32). However, some clinicians recommend that CNS imaging should be a routine assessment or that the interval time between regular examinations in high-risk patients should be shorter $(30,33,34)$. In our study, patients younger than 35 years, positive lymph node involvement, and Her2-positive or triple negative breast cancer had a higher risk of DM. Thus, an intensive surveillance plan should be tailored to these patients. Increasing the frequencies of diagnostic tests in high-risk patients and reducing the frequencies in low-risk patients may improve the efficiency of surveillance. This present model could be a valuable tool in the design of risk-stratified clinical followup studies and could improve surveillance plans.

It is known that nomograms contain more prognostic variables than the traditional TNM staging system (35). Nomograms have been accepted as useful alternative tools for making an accurate prediction through an easy-to-use scoring system. Independent prognostic factors such as age, histology and hormone receptor status could significantly contribute to the individualized prediction of survival (3-5). Identifying patients with different prognoses may have an effect on treatment regimens or patterns of care. Furthermore, oncologists could utilize this nomogram to select the low-risk and high-risk DM patients and provide more appropriate adjuvant treatment and surveillance. For example, high-risk patients should receive more intensive therapies and follow-up, especially those younger than 35 .

Our nomogram demonstrated good accuracy for Chinese individualized survival prediction, and may facilitate the communication between physicians and patients. However, there are some limitations to our study. First, different institutions may have different protocols for breast cancer treatments and surveillance, and selection bias might exist, as this nomogram depended on retrospective cohorts. Second, many breast cancer patients received postoperative comprehensive therapies at outpatient department, however, detailed treatment data were unavailable. Different systemic therapies may affect survival outcomes, and whether incorporating comprehensive therapy strategies into our model would improve its performance is unclear. Third, the classification of breast cancer based on the subtypes is somewhat controversial (36), and the optimal cutoff for ER protein is still being debated (37). A study demonstrated 
that most low ER-staining tumors are HER2-enriched or are basal-like cancer and have similar pathologic features to ER-negative tumors (38). Fourth, several pathological and biological factors, such as urokinase plasminogen activator (uPA)/plasminogen activator inhibitor (PAI-1) (39) and cathepsin D (Cath-D) (40) have been associated with breast tumor aggressiveness and a propensity towards metastasis, but we did not obtain this relevant information. Moreover, this model was constructed only based on Chinese patients; thus, the applicability of this nomogram needs to be validated in other Asian and western populations with a longer follow-up.

\section{Conclusions}

In conclusion, we established and externally validated a precise nomogram for predicting DMFS in non-metastatic breast cancer patients. This convenient model may help physicians to identify subgroups of patients who are in need of specific treatment strategies and intensive surveillance plans.

\section{Acknowledgments}

Funding: This work was supported by grants from the Natural Science Foundation of China (81702615), from the Science and Technology Programme of Guangzhou (20170402009), from the Key Laboratory of Malignant Tumour Molecular Mechanism and Translational Medicine of the Guangzhou Bureau of Science and Information Technology [(2013)163], from the Key Laboratory of Malignant Tumour Gene Regulation and Target Therapy of Guangdong Higher Education Institutes (KLB09001), and from the Guangdong Science and Technology Department (2015B050501004).

\section{Footnote}

Conflicts of Interest: The authors have no conflicts of interest to declare.

Ethical Statement: The authors are accountable for all aspects of the work in ensuring that questions related to the accuracy or integrity of any part of the work are appropriately investigated and resolved. This study was approved by the Institutional Ethics Committees of the SYSMH and GGH (SYSEC-KY-KS-2019-073). Informed consent was waived due to the study's retrospective nature.

\section{References}

1. Fan L, Strasser-Weippl K, Li JJ, et al. Breast cancer in China. Lancet Oncol 2014;15:e279-89.

2. Barral M, Auperin A, Hakime A, et al. Percutaneous Thermal Ablation of Breast Cancer Metastases in Oligometastatic Patients. Cardiovasc Intervent Radiol 2016;39:885-93.

3. Johansson ALV, Trewin CB, Hjerkind KV, et al. Breast cancer-specific survival by clinical subtype after 7 years follow-up of young and elderly women in a nationwide cohort. Int J Cancer 2019;144:1251-61.

4. Poirier É, Desbiens C, Poirier B, et al. Characteristics and long-term survival of patients diagnosed with pure tubular carcinoma of the breast. J Surg Oncol 2018;117:1137-43.

5. Dong G, Wang D, Liang X, et al. Factors related to survival rates for breast cancer patients. Int J Clin Exp Med 2014;7:3719-24.

6. Cardoso F, Costa A, Norton L, et al. 1st International consensus guidelines for advanced breast cancer (ABC 1). Breast 2012;21:242-52.

7. Shen $W$, Shen $Y$, Tan L, et al. A nomogram for predicting lymph node metastasis in surgically resected T1 esophageal squamous cell carcinoma. J Thorac Dis 2018;10:4178-85.

8. Zheng D, Wang Y, Li Y, et al. Predicting prognosis of post-chemotherapy patients with resected IIIA non-small cell lung cancer. J Thorac Dis 2018;10:4186-94.

9. Bogani G, Tagliabue E, Ditto A, et al. Assessing the risk of pelvic and para-aortic nodal involvement in apparent early-stage ovarian cancer: A predictors- and nomogrambased analyses. Gynecol Oncol 2017;147:61-5.

10. Chen LL, Nolan ME, Silverstein MJ, et al. The impact of primary tumor size, lymph node status, and other prognostic factors on the risk of cancer death. Cancer 2009; 115:5071-83.

11. Wishart GC, Azzato EM, Greenberg DC, et al. PREDICT: a new UK prognostic model that predicts survival following surgery for invasive breast cancer. Breast Cancer Res 2010;12:R1.

12. Candido Dos Reis FJ, Wishart GC, Dicks EM, et al. An updated PREDICT breast cancer prognostication and treatment benefit prediction model with independent validation. Breast Cancer Res 2017;19:58.

13. Rudloff U, Jacks LM, Goldberg JI, et al. Nomogram 
for predicting the risk of local recurrence after breastconserving surgery for ductal carcinoma in situ. J Clin Oncol 2010;28:3762-9.

14. Kindts I, Laenen A, Peeters S, et al. Validation of the Web-Based IBTR! 2.0 Nomogram to Predict for Ipsilateral Breast Tumor Recurrence After BreastConserving Therapy. Int J Radiat Oncol Biol Phys 2016;95:1477-84.

15. Jacobs TW, Gown AM, Yaziji H, et al. Specificity of HercepTest in determining HER-2/neu status of breast cancers using the United States Food and Drug Administration-approved scoring system. J Clin Oncol 1999; 17:1983-7.

16. Wiesner FG, Magener A, Fasching PA, et al. Ki-67 as a prognostic molecular marker in routine clinical use in breast cancer patients. Breast 2009;18:135-41.

17. Lu WL, Li HX, Qian BY, et al. The clinical characteristics and prognosis of Chinese early stage breast cancer patients: a retrospective study. Breast J 2010;16:331-3.

18. Lin Z, Yan S, Zhang J, et al. A Nomogram for Distinction and Potential Prediction of Liver Metastasis in Breast Cancer Patients. J Cancer 2018;9:2098-106.

19. Ye FG, Xia C, Ma D, et al. Nomogram for predicting preoperative lymph node involvement in patients with invasive micropapillary carcinoma of breast: a SEER population-based study. BMC Cancer 2018;18:1085.

20. Sorlie T, Tibshirani R, Parker J, et al. Repeated observation of breast tumor subtypes in independent gene expression data sets. Proc Natl Acad Sci U S A 2003;100:8418-23.

21. Smid M, Wang Y, Zhang Y, et al. Subtypes of breast cancer show preferential site of relapse. Cancer Res 2008;68:3108-14.

22. Soni A, Ren Z, Hameed O, et al. Breast cancer subtypes predispose the site of distant metastases. Am J Clin Pathol 2015;143:471-8.

23. Gnant M, Mlineritsch B, Stoeger H, et al. Adjuvant endocrine therapy plus zoledronic acid in premenopausal women with early-stage breast cancer: 62-month followup from the ABCSG-12 randomised trial. Lancet Oncol 2011;12:631-41.

24. Coleman R, de Boer R, Eidtmann H, et al. Zoledronic acid (zoledronate) for postmenopausal women with early breast cancer receiving adjuvant letrozole (ZO-FAST study): final 60-month results. Ann Oncol 2013;24:398-405.

25. Delpech Y, Bashour SI, Lousquy R, et al. Clinical nomogram to predict bone-only metastasis in patients with early breast carcinoma. Br J Cancer 2015;113:1003-9.

26. Kennecke H, Yerushalmi R, Woods R, et al. Metastatic behavior of breast cancer subtypes. J Clin Oncol 2010;28:3271-7.

27. Savci-Heijink CD, Halfwerk H, Hooijer GK, et al. Retrospective analysis of metastatic behaviour of breast cancer subtypes. Breast Cancer Res Treat 2015;150:547-57.

28. Bartsch R, Berghoff AS, Preusser M. Optimal management of brain metastases from breast cancer. Issues and considerations. CNS Drugs 2013;27:121-34.

29. Fontanella C, De Carlo E, Cinausero M, et al. Central nervous system involvement in breast cancer patients: Is the therapeutic landscape changing too slowly? Cancer Treat Rev 2016;46:80-8.

30. Heitz F, Harter P, Lueck HJ, et al. Triple-negative and HER2-overexpressing breast cancers exhibit an elevated risk and an earlier occurrence of cerebral metastases. Eur J Cancer 2009;45:2792-8.

31. Pieńkowski T, Zielinski CC. Trastuzumab treatment in patients with breast cancer and metastatic CNS disease. Ann Oncol 2010;21:917-24.

32. Minisini AM, Moroso S, Gerratana L, et al. Risk factors and survival outcomes in patients with brain metastases from breast cancer. Clin Exp Metastasis 2013;30:951-6.

33. Heitz F, Rochon J, Harter P, et al. Cerebral metastases in metastatic breast cancer: disease-specific risk factors and survival. Ann Oncol 2011;22:1571-81.

34. Niwińska A, Tacikowska M, Murawska M. The effect of early detection of occult brain metastases in HER2positive breast cancer patients on survival and cause of death. Int J Radiat Oncol Biol Phys 2010;77:1134-9.

35. Touijer K, Scardino PT. Nomograms for staging, prognosis, and predicting treatment outcomes. Cancer 2009;115:3107-11.

36. Schwartz GF, Bartelink H, Burstein HJ, et al. Adjuvant therapy in stage I carcinoma of the breast: the influence of multigene analyses and molecular phenotyping. Breast J 2012;18:303-11.

37. Reisenbichler ES, Lester SC, Richardson AL, et al. Interobserver concordance in implementing the 2010 ASCO/CAP recommendations for reporting ER in breast carcinomas: a demonstration of the difficulties of consistently reporting low levels of ER expression by manual quantification. Am J Clin Pathol 2013;140:487-94.

38. Deyarmin B, Kane JL, Valente AL, et al. Effect of ASCO/ CAP guidelines for determining ER status on molecular subtype. Ann Surg Oncol 2013;20:87-93.

39. Duffy MJ, McGowan PM, Harbeck N, et al. uPA and 
Page 10 of 10

PAI-1 as biomarkers in breast cancer: validated for clinical use in level-of-evidence-1 studies. Breast Cancer Res 2014;16:428.

40. Zhang C, Zhang M, Song S. Cathepsin D enhances

Cite this article as: Wang Y, Yang Y, Chen Z, Zhu T, Wu J, Su F, Deng H. Development and validation of a novel nomogram for predicting distant metastasis-free survival among breast cancer patients. Ann Transl Med 2019;7(20):537. doi: 10.21037/ atm.2019.10.10
Wang et al. Nomogram to predict breast cancer survival

breast cancer invasion and metastasis through promoting hepsin ubiquitin-proteasome degradation. Cancer Lett 2018;438:105-15. 